\title{
VERT: a semantic approach for content search and content extraction in XML query processing
}

\author{
Huayu Wu, Tok Wang Ling, and Bo Chen \\ School of Computing, National University of Singapore \\ 3 Science Drive 2, Singapore 117543 \\ \{wuhuayu, lingtw, chenbo\}@comp.nus.edu.sg
}

\begin{abstract}
Processing a twig pattern query in XML document includes structural search and content search. Most existing algorithms only focus on structural search. They treat content nodes the same as element nodes during query processing with structural joins. Due to the high variety of contents, to mix content search and structural search suffers from management problem of contents and low performance. Another disadvantage is to find the actual values asked by a query, they have to rely on the original document. In this paper, we propose a novel algorithm Value Extraction with Relational Table (VERT) to overcome these limitations. The main technique of $V E R T$ is introducing relational tables to store document contents instead of treating them as nodes and labeling them. Tables in our algorithm are created based on semantic information of documents. As more semantics is captured, we can further optimize tables and queries to significantly enhance efficiency. Last, we show by experiments that besides solving different content problems, $V E R T$ also has superiority in performance of twig pattern query processing compared with existing algorithms.
\end{abstract}

\section{Introduction}

XML plays an important role in information exchange nowadays. As a result, how to process queries over XML documents efficiently attracts lots of research interests. In most XML query languages (see, e.g. [2][3]), the queries are expressed as twig patterns. Finding all appearances of a twig pattern in an XML document is a most significant operation in XML query processing.

Normally an XML query includes structural search and content search. E.g. in a query

$$
\text { book }[\text { author }=\text { "Jack" }] / \text { title }
$$

book[author $] /$ title is a structural search and author = "Jack" is a content search. Most of existing works only focus on processing structural search efficiently and very few of them pay high attentions on contents. Content problems include how to properly manage contents, how to efficiently process content search during query processing and how to extract contents to answer the queries. 
E.g. TwigStack [4] and its subsequent algorithm TwigStackList [9] are optimal for processing path and twig pattern queries, but they all suffer from content problems when they process queries with content predicates, because they treat contents the same as other element nodes and perform structural search for the whole query. Although some algorithms like [12] and [11] use subsequence matchings to avoid problems on content search, they still suffer from other problems such as cost for content result fetching in XML databases.

In this paper, we propose a new algorithm to solve different kinds of content problems in twig pattern query processing. Our contribution can be summarized as follows:

- We propose a new algorithm, namely Value Extraction with Relational Table (VERT). Our approach combines value contents to related element or attribute nodes and only assigns label to the element or attribute. Instead of organizing tremendous number of streams for different contents, e.g. streams for value content '33', 'Gehrke' and so on for document in Fig. 1(a), we adopt relational tables to store value contents together with their associated elements or attributes. In this point of view, we can reduce the number of labeled nodes and also we can solve the management problem of contents raised in previous algorithms.

- Content search can be accomplished efficiently by $S Q L$ queries on corresponding relational tables with proper indexes. Furthermore, after finding the appearances of a twig pattern we can easily get the desired value contents from tables. As a result, our approach need not consider the document storage for final results.

- Relational tables are created based on semantic information captured in documents. As more semantics is captured, we can further optimize the tables and queries to achieve better performance, as shown in Section 5.

- Besides solving content problems, we also present experimental results to show the superiority of $V E R T$ and subsequent optimizations over previous algorithms in performance of twig pattern query processing.

The rest of the paper is organized as follows. We first describe some related works in Section 2. After that we discuss background knowledge and motivation for our work in Section 3. The VERT algorithm with two semantics based optimizations is presented in Section 4. We present the experimental results in Section 5 and conclude our work in Section 6.

\section{Related Work}

In early work, Zhang et al. [14] proposed a multi - predicatemergejoin algorithm based on (DocId, Start, End, Level) containment labeling of a XML document. Later an improved stack-based structural join algorithm is proposed 
by Al-Khalifa et al. [1]. These two algorithms, as well as most of prior works decomposed a twig pattern into a set of binary relationships, e.g. parent-child and ancestor-descendant relationships. Then twig pattern matching could be done by matching each binary relationship and combining these basic binary matches. The main problem of such approaches is that intermediate result size may be very large, even when the input and final result sizes are more manageable. To overcome this limitation, Bruno et al. [4] proposed a holistic twig join algorithm, TwigStack, which could avoid producing a large size of intermediate result. However, this algorithm is only optimal for a twig pattern with only ancestor-descendent relationships. There are many subsequent works [9] [8] [5] [10] [7] [13] to optimize TwigStack in terms of I/O, or extend TwigStack for different kinds of problems. In particular, Lu et al. [9] introduced a list structure to make it optimal for queries containing parent-child relationships. However, all these existing works only focus on structural search. For content search they either treat content node the same as element node, or consider how contents are stored and perform a separate operation on content search. ViST [12] and PRIX [11] transform twig pattern queries into sequences and perform subsequence matchings for query processing. They can solve problems on content search, but they still do not pay attention to fetching content results of twig pattern queries, and are not efficient in structural search comparing with TwigStack.

\section{Background and Motivations}

\subsection{Data model and twig pattern}

XML documents are commonly modelled as ordered trees, in which tree nodes represent tags, attributes or text values, and edges represent element-subelement, element-attribute, element-content or attribute-value pairs. We call these binary relationships parent-child relationships (denoted by "/"). Queries normally appear to be twig patterns. A twig pattern is a small tree whose nodes stand for tags, attributes or contents in a document. Different from the parent-child relationship in XML tree, edges in twig query can also be ancestor-descendent relationships (denoted by "//") which stand for that some other nodes may appear on the path between the two nodes connected by "//".

Given a twig pattern query Q, finding all the occurrences in an XML tree T is the main operation for query processing. A match of $\mathrm{Q}$ in $\mathrm{T}$ is identified by a mapping of nodes and edges from $\mathrm{Q}$ to $\mathrm{T}$ such that query node predicates are satisfied by corresponding nodes in the document and the relationships between query nodes are satisfied by corresponding relationships between nodes in the document.

\subsection{Motivations}

TwigStack and its supplementary works are optimal for twig query processing in many cases. In this section, we take TwigStack as an example and discuss 
some drawbacks of existing algorithms regarding to contents, which motivate our research.

Similar as most existing algorithms, TwigStack processes content search in the same way as structural search. The problems regarding to contents in TwigStack can be summarized as follows:

1. In TwigStack, all the nodes including elements, attributes and contents in an XML tree are labeled and the labels are organized in streams. When we build streams for contents, stream management is a problem. Consider a bookstore document shown in Fig. 1(a). There are a large number of books and each of them has a unique ISBN number. For each ISBN number there is a stream, e.g. a stream for '0-07-123057-2', another stream for '0-07-1246509' and so forth. The problem is how to manage the tremendous number of streams. When a query in Fig. 1(b) is issued, it is time consuming to get all the streams with numeric names which are greater than 15 . Although we can organize streams using $B^{+}$tree, after finding all the corresponding streams, to combine labels in them by document order is also time consuming.

2. Streams for contents do not have semantic meanings. This may cause additional checking. When the query is interested in books with price of 20 , structural search scans the stream $T_{20}$. Since in $T_{20}$ we do not differentiate price and quantity, we need check all the labels in this stream though many of them stand for quantity and definitely do not contribute to final answer.

3. When we issue a query over an XML document, what we need is not all the twig pattern appearances represented as tuples of labels, but the content results of that query. Like in the query example above, after finding a certain number of twig pattern appearances which contain element ISBN and title, we need to find their actual values. Since value contents are stored as stream names and it is not practical to get them using labels, they have to move into the document again. That is relevant to how to store and manage XML document and is not negligible.

Our motivation is to avoid all these content problems raised in existing algorithms. After that, twig pattern queries can be processed more efficiently not only in content search, but also in entire execution.

\section{VERT Algorithm}

Some elements or attributes in XML documents describe certain properties of their parent elements, e.g. title, price are properties of book in the bookstore document. We use term property for such element or attribute, and term object for the element described by property. In this section, we first present $V E R T$ algorithm to handle content problems and improve efficiency on content search. Tables in VERT store relationships between properties and their values. Then in Section 4.4 we present another two approaches to optimize $V E R T$ using 


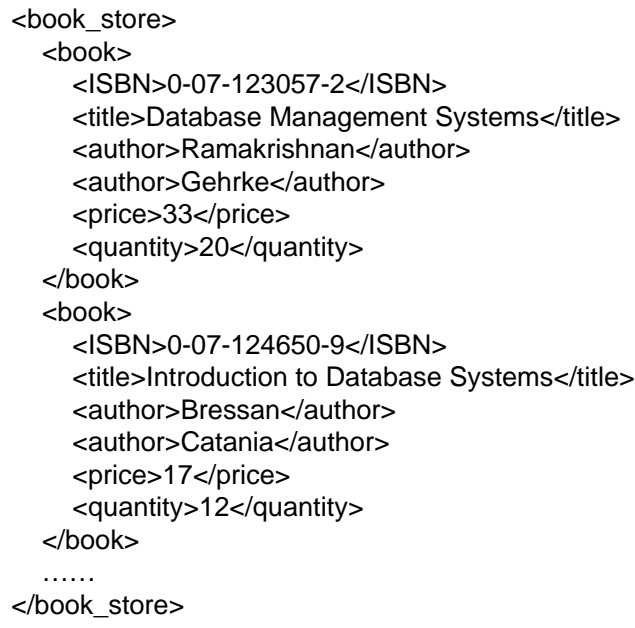

(a) An XML document

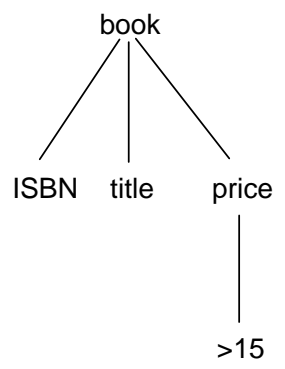

(b) A twig pattern query

Fig. 1. An example of XML document and twig pattern query

semantic information. Tables in these optimizations store relationships between objects and their properties with values.

\subsection{XML document parsing in VERT}

In our first approach, we use tables to store labels of properties and their values. When we parse an XML document, we only label elements and attributes, and put these labels into corresponding streams in document order. Contents in document are not labeled, instead we put them in relational tables together with labels of their property nodes. We adopt interval encoding labeling scheme in our approach (see [6]). The detailed algorithm Parser is presented as follows.

There are three major steps when we parse an XML document: labeling the elements, constructing streams for different types of elements or attributes, and inserting each pair of property and value content into relational tables. $S A X$ reads the documents to transform each tag and content into event and line 3 captures the next event if there are more events in SAX stream. Based on different types of events, different operations are performed accordingly. Line 4-16 are executed if the event $e$ is a starting element. In this case, the first 2 steps are processed. The system first constructs an object for this element and assigns a label to it. It then puts the label into the stream for that tag. A stack $S$ is used to temporarily store the object so that when an ending tag is reached, the system can easily know on which object the operation will be executed. At line 9-14, the system analyzes the attributes for this element if any. Based on the same operating steps, it labels the attributes and puts labels into streams. The attribute values are treated in the same way as element contents. Line 17-18 


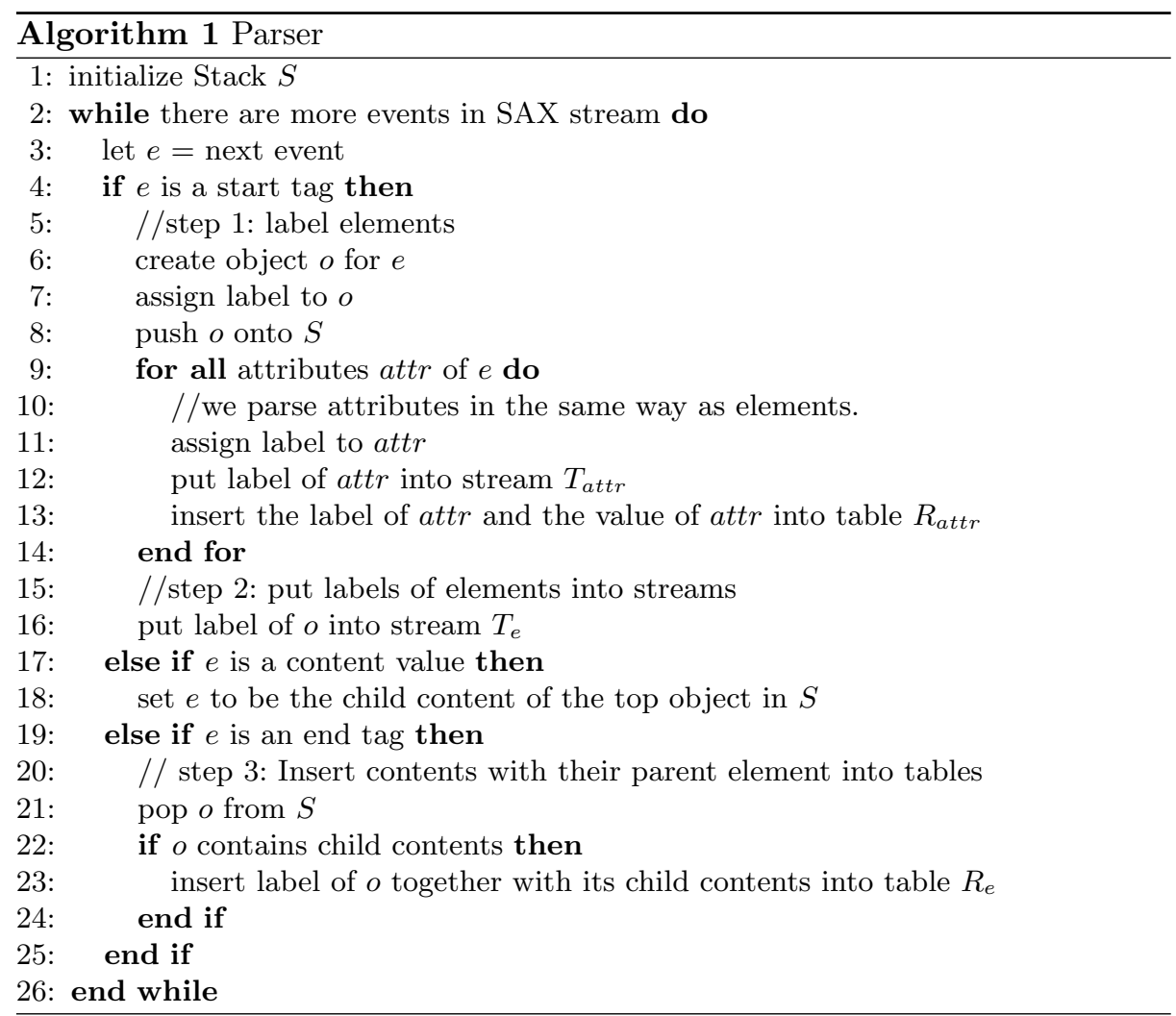

is the case that the event is a content type. Then the content is simply bound to the top object in $S$ for further insertion used. When the event is an ending element in line 19-25, last step is processed, which is popping the top object from the $S$ and inserting the label together with contents into the table for that object. A set of example tables are shown in Fig. 2(c). They are property-value tables. The name of the tables are the property names and each table contains two fields, the label of the property node and value content.

Example 1. Consider the XML data shown in Fig. 1(a), Parser assigns labels to tags and the resulting labeled tree is shown in Fig. 2(a). Then all the labels belonging to the same type of element in XML tree will be passed to the same stream by document order as shown in 2(b). The contents in document together with their parent elements will be stored in corresponding relational tables according to the type of parent elements. Fig. 2(c) shows the resulting tables in this example.

\subsection{Query processing with $V E R T$}

Twig pattern query processing involving contents is composed of two parts. First we analyze and rewrite the query. During this part, for each leaf node which is 


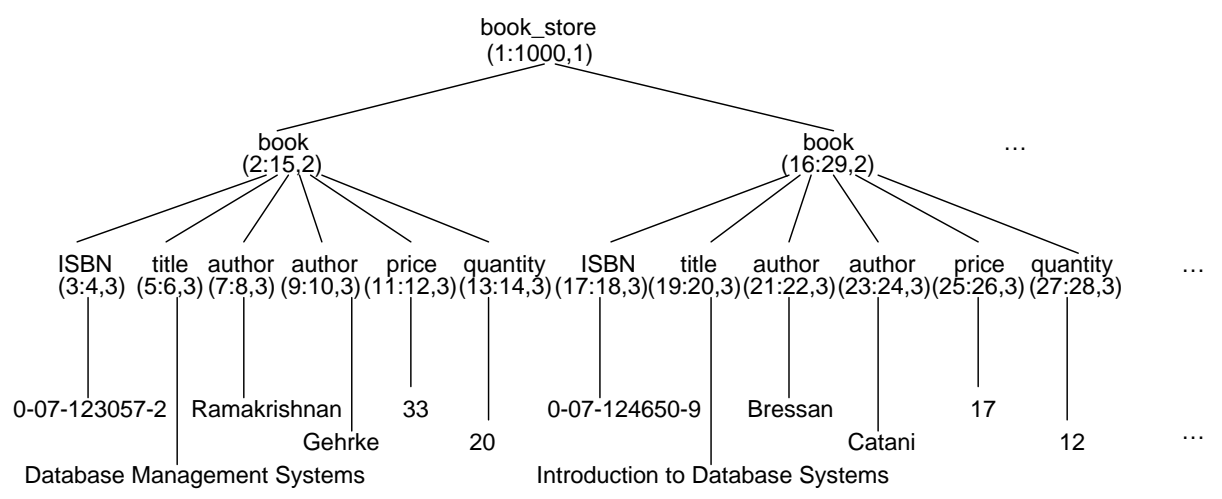

(a) Labeled XML tree

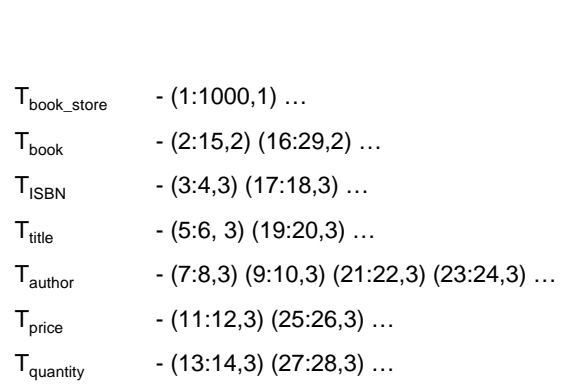

(b) Streams for different elements

\begin{tabular}{|c|c|c|c|c|c|c|}
\hline \multicolumn{2}{|l|}{$\mathrm{R}_{\mathrm{ISBN}}$} & \multicolumn{5}{|l|}{$\mathrm{R}_{\text {title }}$} \\
\hline Label & Content & \multicolumn{2}{|l|}{\begin{tabular}{|l|} 
Label \\
\end{tabular}} & \multicolumn{3}{|c|}{ Content } \\
\hline$(3: 4,3)$ & $0-07-123057-2$ & \multirow{2}{*}{\multicolumn{2}{|c|}{$(5: 6,3)$}} & \multirow{2}{*}{\multicolumn{3}{|c|}{$\begin{array}{l}\text { Database } \\
\text { Management Systems }\end{array}$}} \\
\hline$(17: 18,3)$ & $0-07-124650-9$ & & & & & \\
\hline$\ldots$ & $\ldots$ & \multicolumn{2}{|c|}{$(19: 20,3)$} & \multicolumn{3}{|c|}{$\begin{array}{l}\text { Introduction to } \\
\text { Database Systems }\end{array}$} \\
\hline \multicolumn{2}{|l|}{$\mathrm{R}_{\text {author }}$} & \multicolumn{2}{|l|}{$\ldots$} & \multicolumn{3}{|l|}{$\ldots$} \\
\hline Label & Content & \multirow{2}{*}{\multicolumn{3}{|c|}{$\mathrm{R}_{\text {price }}$}} & \multirow{2}{*}{\multicolumn{2}{|c|}{$R_{\text {quantity }}$}} \\
\hline$(7: 8,3)$ & Ramakrishnan & & & & & \\
\hline$(9: 10,3)$ & Gehrke & \begin{tabular}{|l|} 
Label \\
\end{tabular} & \multicolumn{2}{|c|}{ Content } & Label & Content \\
\hline$(21: 22,3)$ & Bressan & $(11: 12,3)$ & \multicolumn{2}{|l|}{33} & $(13: 14,3)$ & 20 \\
\hline$(23: 24,3)$ & Catani & $(25: 26,3)$ & \multicolumn{2}{|l|}{17} & $(27: 28,3)$ & 12 \\
\hline$\ldots$ & ... & $\ldots$ & \multicolumn{2}{|l|}{$\ldots$} & $\ldots$ & $\ldots$ \\
\hline
\end{tabular}

(c) Tables to store contents

Fig. 2. An example of labeled XML tree with resulting streams and tables by Parser

a value content, a new stream for its parent property node is constructed using the table of that property. In the second part, we process the new query using existing efficient algorithms, e.g. TwigStack in new searching space.

In the main algorithm $V E R T$, we first check for the validity of a given query in line 2-4. Validity of a query $Q$ is defined as whether $Q$ is meaningful for processing. Intuitively this validation can be accomplished by checking whether all the content comparisons in query predicates have parent element. If there is some content comparison in $Q$ appearing in ancestor-descendance relationship ('//') with an element, we consider $Q$ is not meaningful and our algorithm rejects such $Q$. Example 2 shows an invalid query. Line 6-12 recursively handle all the content comparisons in two phases: creating new streams and rewriting the predicates. In detail, Line 7-10 execute $S Q L$ selection on corresponding tables based on the content comparison, and then put all the selected labels, which are satisfied with the content comparison, into the new streams. Line 11 rewrites the query in such a way that the content and their respective parent elements or attributes are replaced by a new element which has an identical name as the corresponding new stream. At the end of the algorithm, we use TwigStack or other efficient algorithm to process the new query with new streams. 


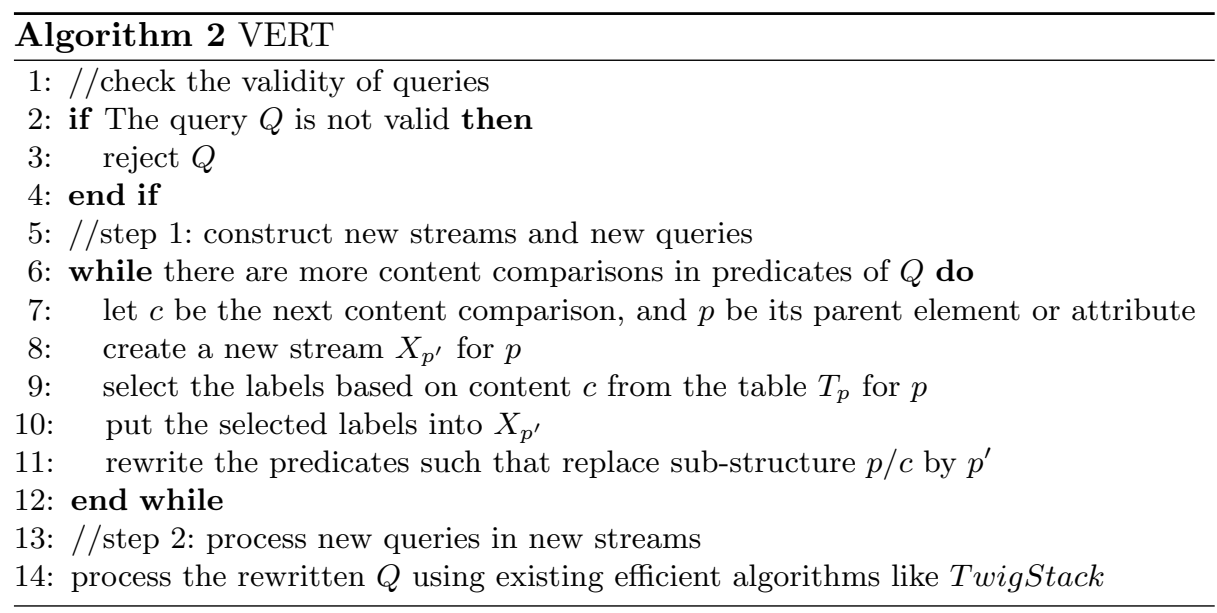

Example 2. Consider the twig pattern query in Fig. 3(a). The value node with content comparison ' $>15$ ' only has an ancestor instead of a parent. In this case we are not sure whether the query wants to get the books with price greater than 15 , or with the quantity greater than 15 . As a result, this twig pattern query is considered invalid and by the line $2-4, V E R T$ rejects this query.

Example 3. The twig pattern query in Fig. 3(b) is valid. By VERT we first find the predicate with content comparison, price $>15$ in this case. In line 7-10, we execute $S Q L$ in table $R_{\text {price }}$ to get all the labels of element price having value content greater than ' 15 '. Since all the records in database are inserted in document order, we can directly add resulting labels into a new stream $T_{\text {price' }}$, which contains all the labels for price with value greater than 15 . Then we rewrite the twig pattern query where the substructure with node price and its child node ' $>15$ ' is replaced by price'. To clearly explain price' in the new query, we use price $>15$ in Fig. 3(c). Finally we process TwigStack on the new query using $T_{\text {price' }}$ for node price $_{>15}$.

\subsection{Analysis of VERT}

In this section, we will analyze our algorithm in four points of view: the management of data including labeled nodes and streams, content extraction for both predicates and final results, the size of streams to be searched and the number of structural joins during query processing.

Data management $V E R T$ combines contents and their parent elements together, and avoids labeling content nodes separately. Suppose an XML document is a full tree with height of $h$ and each element has $k$ children on average. Then the number of labeled elements is $\left(k^{h-1}-1\right) /(\mathrm{k}-1)$ and the number of contents is $k^{h-2}$. When the document is large, the proportion of contents to the sum of elements and contents is around $(k-1) /(2 \mathrm{k}-1)$. In 


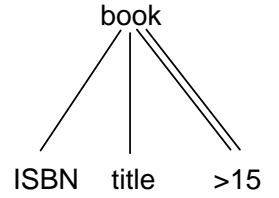

(a) Invalid query

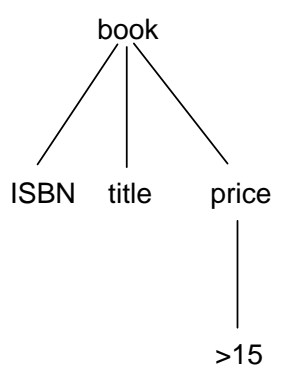

(b) Valid query

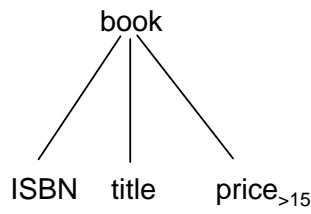

(c) Rewritten query of (b)

Fig. 3. Invalid and valid twig pattern queries

our algorithm, since contents are not labeled, the number of labeled nodes in memory will be reduced nearly by half for large documents and then the size of each stream will also be significantly narrowed down. Furthermore, since large variety of contents are ignored, the number of streams for different types of labeled nodes is limited to the number of element types. So the management of tremendous number of streams in previous work as mentioned in Section 3 can be solved.

Value extraction Consider the XML document in Fig. 1(a) and a query in Fig. 3(b). When we extract the content ' 15 ' to answer this query, in previous approaches we need to move into the stream for content ' 15 '. However, the stream for ' 15 ' contains different semantic labels like the price of a book and the quantity of a book. To mix them together will cause unnecessary search. Instead of searching in streams, $V E R T$ handles contents in semantic tables. In this case, we just move into the table for price and avoid searching for content ' 15 ' under quantity. Furthermore, after getting all the appearances of $I S B N$ and title tags which satisfy the constraint, we aim to find the value contents under these tags. Previous approaches have to move into document again to fetch them because the streams for such contents cannot contribute to final result extraction. This depends how XML documents are stored and is not negligible. VERT can be very efficient to get the desired content results without considering document storage because all the contents are stored in tables instead of streams and we can directly get these contents through $S Q L$ operations on tables. As a result, relational tables are not only helpful in content search, but also usable to get desired contents based on the labels found.

Stream searching reduction Pre-processing contents is essential to reduce the size of streams. Consider the query that we want to find the quantity for a book with 'ISBN $=0-07-123057-2$ ' on the bookstore document. If the number of different books is $b$, the size of stream for element $I S B N$ is also $b$ in previous approaches, as shown in Fig. 4(a). Then we need 
$O(b)$ to scan all the labels in $I S B N$ stream. $V E R T$ processes selection in advance, such that the new stream for $I S B N$ is created based on content '0-07-123057-2'. That means the new stream has only 1 label inside since $I S B N$ is the key for books. Fig. 4(b) shows the rewritten query and size of new stream. $T_{I S B N^{\prime}}$ is the new stream for element $I S B N$, and in Fig. 4(b) we use $I S B N_{0-07-123057-2}$ to explain $I S B N^{\prime}$. So when the selectivity of an element is high, like in this example, $V E R T$ also has high superiority to previous algorithms because it significantly reduces the searching in stream.

Structural joins reduction There are two factors driving the high performance of $V E R T$. One is searching space reduction as mentioned above and the other factor is number of structural joins reduction. Still consider the example in Fig. 4. The rewritten query has only two parent-child relationships need structural joins, while the original query has three. As we know structural join is an expensive operation, the reduction of structural joins leads a higher performance. Optimizations to further reduce size of streams and number of structural joins will be discussed in next section.

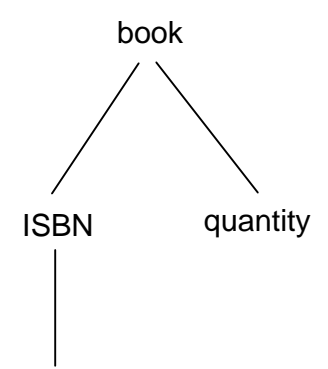

$0-07-123057-2$ (a) Original query with corresponding stream size $\left|T_{b o o k}\right|=\mathrm{b}$ and $\left|T_{I S B N}\right|=\mathrm{b}$

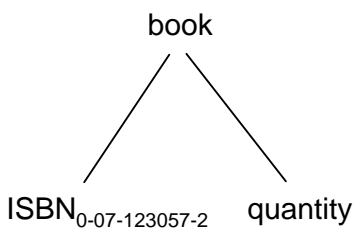

(b) Rewritten query for (a) with corresponding stream size $\left|T_{\text {book }}\right|=\mathrm{b}$, and stream size for node $I S B N_{0-07-123057-2}=\left|T_{I S B N^{\prime}}\right|=1$

Fig. 4. Original and rewritten query examples in VERT

\subsection{Optimizations for VERT}

Tables in $V E R T$ store label of each property and its value content. This approach differentiates contents by properties. However, there are still more semantics can be captured. With more semantic information, we can improve the performance by further reducing size of streams and number of structural joins in most documents. This motivates two approaches of optimizations for VERT. 
Observation 1: Generally, after knowing the value on a certain property of an object, most queries want to find that object and then get some other properties of that object. For the query in Fig. 4, with the $I S B N$ value we need to find corresponding book and get the quantity of that book. After $V E R T$ rewriting the query, the size of stream for $I S B N$ is significantly reduced. However the size of stream for book is still $b$, which means we need to search all the $b$ labels in book stream although we know there is only one matches with the label in $I S B N$ stream. As a result, we can further rewrite the query to get the object book directly from property $I S B N$ value.

Optimization 1: Instead of storing labels of property nodes and their value contents, we can put the labels of objects with property values into tables. E.g. in the bookstore document we put value contents for $I S B N$, title and so forth with labels of corresponding book in object/property tables as shown in Fig. $5(a)$. The 'Label' field of each table stores the label of object book and the following 'Content' corresponds the value contents of different properties in different tables, e.g. in $R_{b o o k / I S B N}$ 'Content' in each tuple is the $I S B N$ value of the book with 'Label' in the same tuple. The query in Fig. 4(a) is rewritten accordingly, as shown in Fig. 5(b), where $T_{b o o k^{\prime}}$ is the new stream for element book and book ${ }_{I S B N=0-07-123057-2}$ is to explain book $k^{\prime}$. In new tables, we can directly select the label for book based on ISBN number in $R_{b o o k / I S B N}$ without considering tags for element $I S B N$. Now we not only reduce the size of $T_{b o o k}$, but also reduce the number of structural joins to be 1 . So we can get a higher performance when we execute the new query.

\begin{tabular}{|c|c|c|c|c|c|c|}
\hline \multicolumn{2}{|l|}{$R_{\text {booklISBN }}$} & \multicolumn{5}{|c|}{$\mathrm{R}_{\text {booktitile }}$} \\
\hline Label & Content & \multicolumn{2}{|l|}{\begin{tabular}{|l|} 
Label \\
\end{tabular}} & \multicolumn{3}{|c|}{ Content } \\
\hline$(2: 15,2)$ & $0-07-123057-2$ & \multirow{2}{*}{\multicolumn{2}{|c|}{$(2: 15,2)$}} & \multirow{2}{*}{\multicolumn{3}{|c|}{$\begin{array}{l}\text { Database } \\
\text { Management Systems }\end{array}$}} \\
\hline$(16: 29,2)$ & 0-07-124650-9 & & & & & \\
\hline$\ldots$ & $\ldots$ & \multicolumn{2}{|c|}{$(16: 29,2)$} & \multicolumn{3}{|c|}{$\begin{array}{l}\text { Introduction to } \\
\text { Database Systems }\end{array}$} \\
\hline \multicolumn{2}{|l|}{$\mathrm{R}_{\text {book/author }}$} & & \multicolumn{3}{|c|}{ 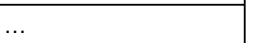 } \\
\hline Label & Content & \multirow{2}{*}{\multicolumn{3}{|c|}{$\mathrm{R}_{\text {book/price }}$}} & \multirow{2}{*}{\multicolumn{2}{|c|}{$\mathrm{R}_{\text {bookkquantity }}$}} \\
\hline$(2: 15,2)$ & Ramakrishnan & & & & & \\
\hline$(2: 15,2)$ & Gehrke & Label & \multicolumn{2}{|c|}{ Content } & Label & Content \\
\hline$(16: 29,2)$ & Bressan & $(2: 15,2)$ & \multicolumn{2}{|l|}{33} & $(2: 15,2)$ & 20 \\
\hline$(16: 29,2)$ & Catani & $(16: 29,2)$ & \multicolumn{2}{|l|}{17} & $(16: 29,2)$ & 12 \\
\hline$\ldots$ & $\ldots$ & $\ldots$ & \multicolumn{2}{|l|}{$\ldots$} & $\ldots$ & $\ldots$ \\
\hline
\end{tabular}

(a) Tables in VERT optimization 1

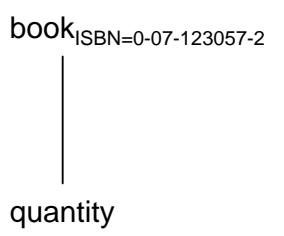

(b) Rewritten query for Fig 4(a) with stream size of book $_{I S B N=0-07-123057-2}=$ $\left|T_{\text {book }}\right|=1$

Fig. 5. Tables and queries in VERT optimization 1

However, this optimization may lose order information in some cases. If we want to get all the authors' names of a certain book, since we ignore the element 
author and get all the name contents from $R_{\text {book/author }}$, we cannot differentiate the order in document. This limitation can be solved by adding ordinal number to different contents if the order is important.

Observation 2: There are some queries with multiple predicates on a certain element. E.g. a query on the bookstore document: find the ISBN number of the book with title 'Database Management Systems' and price of 33. To answer this query, $V E R T$ with optimization 1 needs to find the books with title 'Database Management Systems' and books with price of 33 separately and join them to get results. With semantic information, we know title and price are properties of object book. If we have a table for this object which contains both of the properties, books satisfying these two constraints can be found directly and intermediate results can be avoided.

Optimization 2: A simple idea is to pre-merge tables in optimization 1 based on the same objects. But for multi-value properties, like author in our example, it is not practical to merge it with other properties. The information on multi-value properties can be found in document schema. After knowing this, we can merge all the single-value properties of an object into one table and keep tables for multi-value properties remain as what they are in optimization 1 . The resulting tables for bookstore document is shown in Fig. 6. In $R_{b o o k}$, each label of book is stored with all the single-value property contents of that book. When we process queries with multiple predicates on a certain object, we can do selection in that object table using these predicate constraints in one time. In this way, we can even simplify the query and prune intermediate results.

$\mathrm{R}_{\text {book }}$
\begin{tabular}{|l|l|l|l|l|}
\hline Label & ISBN & Title & Price & Quantity \\
\hline$(2: 15,2)$ & $0-07-123057-2$ & $\begin{array}{l}\text { Database } \\
\text { Management Systems }\end{array}$ & 33 & 20 \\
\hline$(16: 29,2)$ & $0-07-124650-9$ & $\begin{array}{l}\text { Introduction to } \\
\text { Database Systems }\end{array}$ & 17 & 12 \\
\hline$\ldots$ & $\ldots$ & $\ldots$ & $\ldots$ & $\ldots$ \\
\hline
\end{tabular}

$\mathrm{R}_{\text {booklauthor }}$
\begin{tabular}{|l|l|}
\hline Label & Author \\
\hline$(2: 15,2)$ & Ramakrishnan \\
\hline$(2: 15,2)$ & Gehrke \\
\hline$(16: 29,2)$ & Bressan \\
\hline$(16: 29,2)$ & Catani \\
\hline$\ldots$ & $\ldots$ \\
\hline
\end{tabular}

Fig. 6. Tables in VERT optimization 2

Declarations: Optimizations of $V E R T$ are based on semantics captured from schema or document, or even declared by document owners. Generally, the more semantic information known, the further our algorithm can be optimized and the better performance can be achieved.

\section{Experiments}

In this section, we present experimental results on the performance of twig pattern search under $V E R T$ algorithms with and without optimizations, which are introduced in section 4, and TwigStack. Final result extraction for each 
query can be done simply by selection in corresponding tables in our approach, however, in other algorithms it depends on the database implementation. The comparison for final result extraction is not included in our experiments.

\subsection{Experimental Settings}

Implementation and Hardware: We implemented all algorithms in Java. The experiments were performed on a $3.0 \mathrm{GHz}$ Pentium 4 processor with $1 \mathrm{G}$ RAM under OS of Windows XP.

XML Data Sets: We used three real-world and synthetic data sets for our experiments: NASA, DBLP and XMark. NASA is a $25 \mathrm{MB}$ document with complex DTD schema. DBLP data set is a 127MB fragment of DBLP database. The characteristic of this data set is simple DTD schema and large data sources. We also used XMark benchmark data with size of 110MB.

Queries: We selected three meaningful queries for each data set. All the queries chosen contain predicates with content comparison, since content predicates appear in most practical queries. Generally, there are three types of queries: queries with predicates on equality comparison, queries with predicates on range comparison and queries with multiple predicates on different comparisons. The queries are shown in Table 1.

\begin{tabular}{|c|c|c|}
\hline Query & Data Set & XPath Expression \\
\hline Q1 & NASA & $\begin{array}{l}\text { //dataset//source/other[/date/year }>^{\prime} 1919 \text { ' and year <'2000']/author } \\
\text { /lastName }\end{array}$ \\
\hline Q2 & NASA & //dataset/tableHead[//field/name='rah']//tableLinks //title \\
\hline Q3 & NASA & $\begin{array}{l}\text { //dataset/history/ingest[/date[/year }>^{\prime} 1949 \text { ' and year }<\text { '2000'] } \\
\text { [/month='Nov'][/day > '14' and day }<\text { '21']]/creator /lastName }\end{array}$ \\
\hline Q4 & $\overline{\text { DBLP }}$ & /dblp/article[/author $={ }^{6} \mathrm{Jim}$ Gray']/title \\
\hline Q5 & DBLP & //proceedings[/year>'1999']/isbn \\
\hline Q6 & DBLP & $\begin{array}{l}\text { //inproceedings[/title='A Flexible Modelling Approach for Software } \\
\text { Reliability Growth'][/year='1987'][/author='Sergio Bittanti'] } \\
\text { /booktitle }\end{array}$ \\
\hline Q7 & XMark & $\begin{array}{l}\text { //regions/africa/item[/mailbox/mail/from='Libero Rive'] } \\
\text { /description }\end{array}$ \\
\hline Q8 & XMark & //item[//mail/date>'Sep']/location \\
\hline Q9 & XMark & $\begin{array}{l}\text { //item[/location='United State'][/mailbox/mail[/date= } \\
\text { '02/11/1999'][/to='Aamer Krolokowski']]/description }\end{array}$ \\
\hline
\end{tabular}

Table 1. Experimental queries 


\subsection{Experimental Results and Analysis}

Our experiments mainly compare the stream management and total execution time between TwigStack and our approaches. The implementation of TwigStack adopts $B^{+}$tree to organize streams, which ensures high performance of content stream management. The number of labeled nodes and number of streams to be managed for the three data sets under the two approaches are shown in Table 2 . This result validates the analysis about the data management in last section.

\begin{tabular}{|l|l|l|l|l|}
\hline \multirow{2}{*}{ Data Set } & \multicolumn{2}{|c|}{ Number of Labeled Nodes } & \multicolumn{2}{c|}{ Number of Streams } \\
\cline { 2 - 5 } & TwigStack & VERT & TwigStack & VERT \\
\hline NASA & 997,987 & 532,963 & 121,833 & 68 \\
\hline DBLP & $6,771,148$ & $3,736,406$ & 388,630 & 37 \\
\hline XMark & $5,215,282$ & $2,048,193$ & 353,476 & 75 \\
\hline
\end{tabular}

Table 2. Number of labeled nodes and streams using TwigStack and VERT

The experimental results of execution time for the three data sets are shown in Fig. 7. From the results, we can see the execution time reduction is significant for all the queries in DBLP document. This is in accord with our analysis in section 4.3, that is our approach works quite well for such XML document that has simple schema but large data sources. In DBLP document, there are only several types of data like proceedings, thesis, articles and so forth. There are large quantity of works under each type. The properties of each work type that appear as sub-elements in document are mostly the same and depth of the data tree is 3. As a result, for DBLP data, when we rewrite the query to reduce the query depth, we prune tremendous number of unnecessary tag checkings. Q2 in NASA data set is another example for the reason why $V E R T$ has higher performance than other approaches. The tag 'name' appears quite frequently in document with different semantic meanings, however, in Q2 what we are interested is only the field name. Instead of scanning all the 'name', our approach can move into field names directly using semantic tables. In this way, the execution time can be significantly reduced.

Comparing with optimization 1 and optimization 2 of $V E R T$, we can see from the experimental result that for single-predicate queries there is no obvious difference. However, for multi-predicate queries, optimization 2 has a better performance as shown in Q3, Q6 and Q9. This again proves our analysis in Section 4.4.

\section{Conclusion and future work}

In this paper, we propose a novel algorithm $V E R T$ to solve different content problems raised in existing algorithms. Unlike TwigStack and its subsequent algorithms, our approach uses semantic tables to do content search, and then 


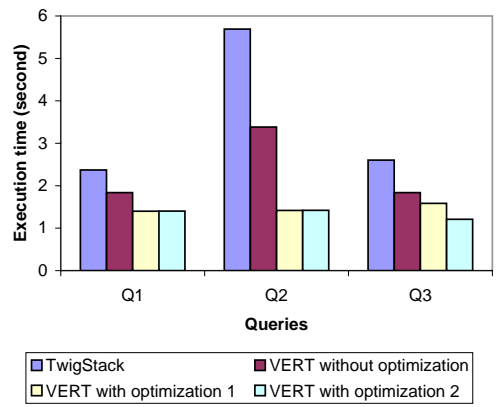

(a) NASA

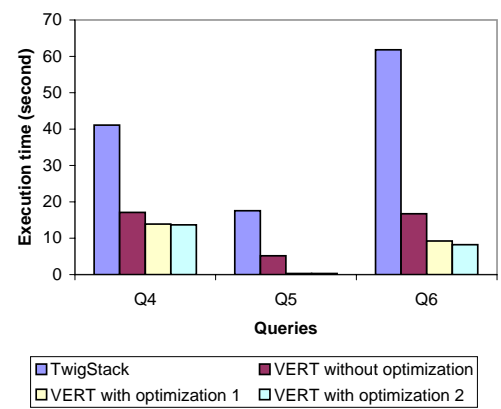

(b) DBLP

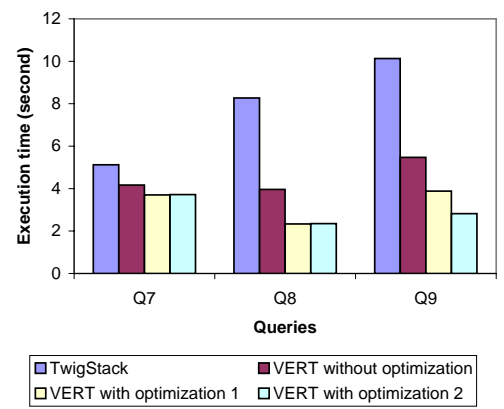

(c) XMark

Fig. 7. Execution time by TwigStack and VERT without optimizations, with optimization 1 and with optimization 2 in three XML documents.

avoids the management of tremendous number data streams. Besides, VERT can efficiently extract contents for predicate comparisons during query processing. Experimental results show that our method is much more efficient than TwigStack for queries with content comparison as predicates. To answer the query, our method need not consider how the document stored in database. Instead, we can directly get the content results from tables.

One direction for future research to improve our algorithm is to discover more semantics in XML document and combine the semantic information into relational tables. Queries can be processed more efficient based on semantic tables and query rewriting by reducing unnecessary searches, number of structural joins and intermediate results. Also, our relational approach gives a new scheme to relate XML query processing algorithms to XML databases.

\section{References}

1. S. Al-Khalifa, H. V. Jagadish, J. M. Patel, Y. Wu, N. Koudas, and D. Srivastava. Structural joins: A primitive for efficient XML query pattern matching. In Proc. of ICDE (2002) 
2. A. Berglund, D. Chamberlin, M. F. Fernandez, M. Kay, J. Robie, and J. Simeon. XML Path Language (XPath) 2.0. W3C Working Draft (2003)

3. S. Boag, D. Chamberlin, M. F. Fernandez, D. Florescu, J. Robie, and J. Simeon. XQuery 1.0: An XML Query. W3C Working Draft (2003)

4. N. Bruno, N. Koudas, and D. Srivastava. Holistic twig joins: Optimal XML pattern matching. In Proc. of ACM SIGMOD (2002)

5. T. Chen, J. Lu, and T. W. Ling. On boosting holism in XML twig pattern matching using structural indexing techniques. In Proc. of SIGMOD Conference (2005)

6. T. Grust. Accelerating XPath location steps. In Proc. of SIGMOD Conference (2002)

7. H. Jiang, H. Lu, and W. Wang. Efficient processing of XML twig queries with OR-predicates. In Proc. of SIGMOD Conference (2004)

8. H. Jiang, W. Wang, H. Lu, and J. Yu. Holistic twig joins on indexed XML documents. In Proc. of VLDB Conference (2003)

9. J. Lu, T. Chen, and T. W. Ling. Efficient processing of XML twig patterns with parent child edges: a look-ahead approach. In Proc. of CIKM (2004)

10. J. Lu, T. W. Ling, C. Chan, and T. Chen. From region encoding to extended dewey: On efficient processing of XML twig pattern matching. In Proc. of VLDB Conference (2005)

11. P. R. Rao and B. Moon. PRIX: Indexing and Querying XML Using Prufer Sequences. In Proc. of ICDE (2004)

12. H. Wang, S. Park, W. Fan, and P. S. Yu. ViST: A Dynamic index method for querying XML data by tree structures. In Proc. of SIGMOD Conference (2003)

13. T. Yu, T. W. Ling, and J. Lu. Twigstacklistnot: A holistic twig join algorithm for twig query with NOT-predicates on XML data. In Proc. of DASFAA (2006)

14. C. Zhang, J. Naughton, D. Dewitt, Q. Luo, and G. Lohman. On supporting containment queries in relational database management systems. In Proc. of ACM SIGMOD (2001) 